\title{
Glazing effects on bread crust and crumb staling during storage.
}

\begin{abstract}
The uses of cornstarch, egg white, skim milk and shortening as glazes on bread surfaces were investigated for its ability to reduce bread staling. Four concentrations of glazes were applied to sweet bun surfaces. Bread crumb and crust samples were measured for its firmness and moisture during storage. The rates of change in firmness and moisture contents of crumb and crust with storage time were analyzed by comparing with nonglazed breads as control. While both crumb and crust firmness and the crust moisture content increased with storage time, crumb moisture content decreased significantly (P PRACTICAL APPLICATIONSThe use of glaze materials in baked products may provide more than an aesthetic function of creating appearance appeal. This research shows that glazing of breads reduces bread staling through its ability to retain crumb moisture and reduce crumb firming. This finding provides means of improving bread quality in terms of textural properties and its shelf life by means of processing and with no alter in ingredients' formulation.
\end{abstract}

Keyword: Glaze; Bread; Crust; Crumb; Storage. 